\title{
Prediction and Modelling of Corrosion in Steel Storage Tank Using Non-destructive Inspection
}

\author{
Mosaad Mohamed Sadawy ${ }^{1}$ and Eltohamy Rabie Elsharkawy ${ }^{2}$ \\ 1. Faculty of Engineering, Al-Azhar University, Cairo, Egypt \\ 2. Nuclear \& Radiological Regulatory Authority, Cairo, Egypt
}

Received: December 01, 2013 / Accepted: December 20, 2013 / Published: December 25, 2013.

\begin{abstract}
The ability to detect and classify damages in complex materials and structures is an important problem from both safety and economical perspectives. This paper develops a novel approach based on geostatistical analysis for the corrosion prediction. Our approach is based on using geostatistics for modeling oil tank thickness from nondestructive data. First of all the semivariogram was obtained from the scatter points set. Then computing the ordinary kriging predictions at unmonitored points. Finally, simulating the thickness along the surface of the tank.
\end{abstract}

Key words: Geostatistical analysis, non-destructive tests, semivariogram, ordinary Kriging, simulation.

\section{Introduction}

Corrosion of pipelines, pressure vessels and storage tanks of any liquid contain radioactive material or oil are a very important issue in the petrochemical and nuclear industries. In these industries the pipelines, pressure vessels and storage tanks are often carrying hazardous or valuable liquids. The environmental and economic impacts of any leakage can have severe consequences. The structures that need inspection are often large and only partially accessible [1-5]. The inspection and maintenance strategies to detect damages can be costly and economical planning is mandatory [2].

During the last years increasingly powerful and versatile geostatistical tools have been developed for geoscience applications. Geostatistics is a set of tools used for characterizing spatial variation, spatial prediction, spatial simulation and spatial optimization (e.g., sampling design). Applications of geostatistics

Corresponding author: Eltohamy Rabie Elsharkawy, assistant professor, research fields: material, non-destructive testing, metal testing, quality, corrosion and metallurgy. E-mail: Tohamy1000@gmail.com. are found in a wide range of fields including biology, environmental science, geography, geology, meteorology and mining [6]. The goal of geostatistical analysis is to predict values where no data have been collected [7].

The semivariogram is a fundamental tool in geostatistics, which measures regionalized spatial variability of $x\left(u_{a}\right)$, where $u_{a}$ is the coordinate vector at each of the observation points $(\mathrm{a}=1,2,3, \ldots N)$ in a two-or three-dimensional space for the variable $x$. The empirical semivariogram $(\gamma(h))$ is defined as half the average quadratic difference between two observations of a variable separated by a distance vector $h$ [8-15]:

$$
\gamma(h)=\frac{1}{2 N(h)} \sum_{a=1}^{N(h)}\left[x\left(u_{a}\right)-x\left(u_{a}+h\right)\right]^{2}
$$

The experimental semivariograms were fitted with various theoretical models like spherical, exponential, gaussian, linear and power by the weighted least square method. The theoretical model that gave minimum standard error is chosen for further analysis. The adequacy of the fitted models was checked on the 
basis of validation tests.

Kriging is a technique of making optimal, unbiased estimates of regionalized variables at unsampled locations using the structural properties of the semivariogram and the initial set of data values. Kriging takes into consideration the spatial structure of the parameter and hence score over other methods like arithmetic mean method, nearest neighbour method, distance weighted method, and polynomial interpolation. Also, kriging provides the estimation variance at every estimated point, which is an indicator of the accuracy of the estimated value. This is considered as the major advantage of kriging over other estimation techniques [9].

Ordinary kriging (OK), allows the mean to vary spatially: the mean is estimated for each prediction neighborhood. OK predictions are weighted averages of the $n$ available data. The OK weights define the Best Linear Unbiased Predictor (BLUP). The OK prediction, $\underset{o k}{\dot{Z}}\left(x_{0}\right)$, is defined as $[11,12]$ :

$$
\underset{o k}{Z}\left(x_{0}\right)=\sum_{n=1}^{n} \lambda_{a}^{o k} Z\left(x_{a}\right)
$$

with the constraint that the weights, $\lambda_{a}^{o k} \mathrm{OK}$, sum to 1 to ensure an unbiased prediction:

$$
\sum_{n=1}^{n} \lambda_{a}^{o k}=1
$$

So, the objective of the kriging system is to find appropriate weights by which the available observations will be multiplied before summing them to obtain the predicted value. These weights are determined using the coefficients of a model fitted to the variogram (or another function such as the covariance function). The kriging prediction error must have an expected value of 0 :

$$
E\left\{\dot{Z}\left(x_{0}\right)-Z\left(x_{0}\right)\right\}=0
$$

The kriging (or prediction) variance $\sigma_{o k}^{2}\left(x_{0}\right)$, is expressed as:

$$
\sigma_{o k}^{2}\left(x_{0}\right)=E\left[\left\{\dot{Z}\left(x_{0}\right)-Z\left(x_{0}\right)\right\}^{2}\right]
$$

$$
\begin{aligned}
=-\gamma(0) & -\sum_{a=1}^{n} \sum_{\beta=1}^{n} \lambda_{a}^{o k} \lambda_{\beta}^{o k} \gamma\left(x_{a}-x_{\beta}\right) \\
& +2 \sum_{a=1}^{n} \lambda_{a}^{o k} \gamma\left(x_{a}-x_{0}\right)
\end{aligned}
$$

That is, we seek the values of $\lambda_{1} \ldots . . . \lambda_{n}$ (the weights) that minimize this expression with the constraint that the weights sum to one (Eq. (3)). This minimization is achieved through Lagrange multipliers. The conditions for the minimization are given by the OK system comprising $n+1$ equation and $n+1$ unknown:

$\left\{\begin{array}{l}\sum_{\beta=1}^{n} \lambda_{\beta}^{o k} \gamma\left(x_{a}-x_{\beta}\right)+\psi_{o k}=\gamma\left(x_{a}-x_{\beta}\right) \quad \mathrm{a}=1, \ldots ., \mathrm{n} \\ \sum_{\beta=1}^{n} \lambda_{\beta}^{o k}=1\end{array}\right.$

where, $\psi_{o k}$ is a Lagrange muliplier. Knowing $\psi_{o k}$, the prediction variance of $\mathrm{OK}$ can be given as:

$$
\sigma_{o k}^{2}=\psi_{o k}-\gamma(0)+\sum_{a=1}^{n} \lambda_{a}^{o k} \gamma\left(x_{a}-x_{0}\right)
$$

The kriging variance is a measure of confidence in predictions and is a function of the form of the variogram, the sample configuration and the sample support (the area over which an observation is made, which may be approximated as a point or may be an area) [13].

Our study is based on using geostatistics analysis for modeling oil tank thickness from nondestructive data. First of all the semivariogram was obtained from the scatter points set to be interpolated. Then computing the ordinary kriging predictions and finally, simulating the thickness along the surface of the tank.

\section{Experiments}

The present study was carried out on crude oil storage tank T-3510A which stores oil before exporting through 12", oil export line to El-hamra terminal, Western desert, Egypt. Settling operation is done through the tank to separate all residual water. $\mathrm{T}$ 3510 A consists of nineteen plates every pate has nine meters long and $2.4 \mathrm{~m}$ width which was arranged 
through the tank shell in three courses. T-3510A with a chemical composition of C $0.21 \%$, Mn $1.5 \%$, S $0.045 \%$ and Fe balance. The ultrasonic measurements were carried out by Krautkramer DM4 at fixed intervals of $1.2 \mathrm{~m} \times 4.5 \mathrm{~m}$ (Fig. 1). The ultrasonic thickness gauge was calibrated before reading by standard calibration block with variable thickness.

Data sets were characterised statistically; this description included the calculation of mean, median, minimum, maximum, coefficient of variation, standard deviation, skewness.

Spatial variability of tank thickness was determined using the geostatistical method for estimating the relationship between the variance and the sampling interval. The resultant data were employed to plot variograms along $(h)$ distance. Kriging was used to compile cartograms of tank thickness. If trends were present, then regression kriging was used. Like regression, regression kriging recognizes that the variation has two components, namely the trend and the residuals from the trend. It differs from regression in that it takes into account the dependence in the residuals, which it treats as spatially correlated stationary random variables. So the residuals have a variogram, and the kriging systems draw their entries from this variograms.

The regression kriging predictions are computed as follow. The first step is to model trend, as in trend-surface analysis, and remove it from the data. The residuals from the trend (or detrended data) are treated as spatially correlated stationary random variables. Their variogram is computed and modeled and then used to krig. Finally, the trend is added back to the kriged estimates and finally simulation .

Histogram were made with (Smith statistical backage,Version 2.8, Copyright (1995-2005 Garey Smith). Calculations and variogram plotting were made with (SURFER,Version 10.0, Copyright (C1993-2010, Golden Software, Inc.). Spatial distribution map and simulation were made with (Gridat Geostatistical software, Version 2.0.1,
Copyright @2010 ampiroid).

\section{Results and Discussion}

\subsection{Statistical Analysis}

The distribution of thickness was checked through building of histogram (Figure 2) and performing of statistical analysis to cheek the type of distribution and homogeneity (Table 1). It is shown from Figure 2 that the distributions is homogenous, i.e. without statistically significant gaps. On the other hand Table 1 indicates that the thickness ranged from 13.9 to 14.8. The mean value of the data sets was 14.4, which was very close to the median value that was 14.5 . The coefficient of skewness is not very high $(-0.52)$, indicating that the distribution is only slightly asymmetric. The very low value of the coefficient of variation (0.017) reflect the fact that the histograms do not have a tail of high values. The ordinary kriging method works better if the distribution of the data values is close to a normal [14]. Therefore, it is interesting to see how close the distribution of the data values comes to being normal. Fig. 2 shows also the plots of the normal distribution adjusted to the

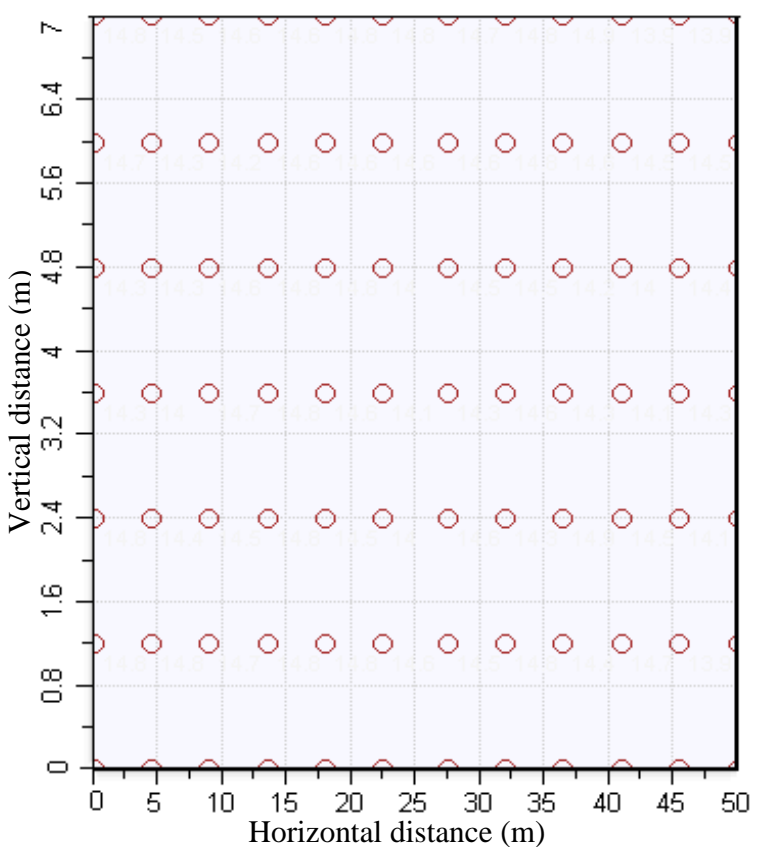

Fig. 1 The ultrasonic reading spot distribution for T-3510A. 


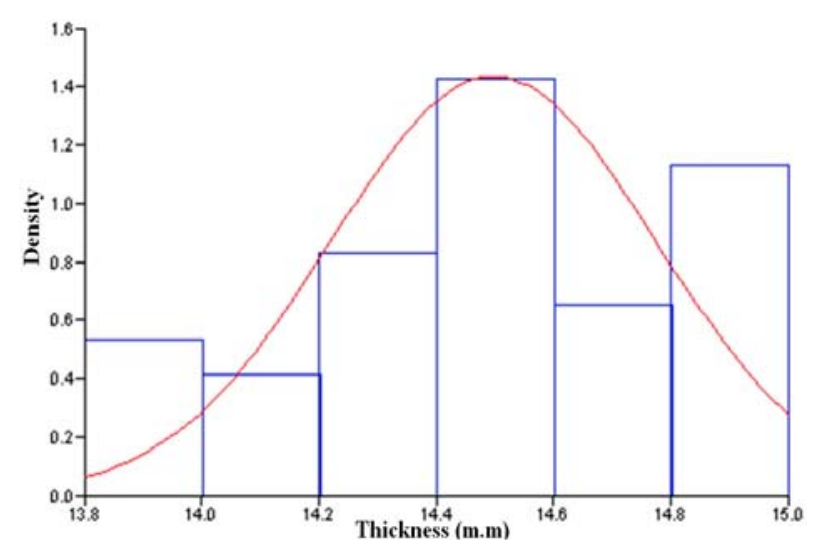

Fig. 2 Histogram of oil storage tank thickness.

Table 1 Summary statistics of oil storage tank thickness measurements.

\begin{tabular}{ll}
\hline Statistical factors & Thickness $(\mathrm{mm})$ \\
\hline Minimum & 13.9 \\
25th quartile & 14.4 \\
Median & 14.5 \\
75th quartile & 14.6 \\
Mean & 14.4 \\
Maximum & 14.8 \\
Coefficient of skewness & -0.62 \\
Coefficient of variation & 0.017 \\
\hline
\end{tabular}

histogram. It can be seen that the histograms are reasonably close to the normal distribution.

\subsection{Spatial Dependence Analysis}

Estimation of the experimental variogram was undertaken for each sample to enable identification of spatial variability in thickness: low thickness zones indicate the presence of corrosive ions or water which allow increasing the electrochemical reaction and hence increasing the corrosion rate. Different types of semivariogram models used to fit the points include exponential, Gaussian, spherical, tetraspherical, pentaspherical, Hole effect models. The spherical model had the best fits and was selected as the theoretical model applied for spatial predictions. The spherical model is defined as [14]:

$$
\gamma(h)= \begin{cases}c_{0}+c\left(\frac{3 h}{2 a}-\frac{1}{2}\left(\frac{h}{3}\right)^{3}\right) & \text { for } h \leq a \\ c_{0}+c & \text { for } h>a\end{cases}
$$

where $\left(c_{0}\right)$ is the nugget variance parameter, $(c)$ the sill variance parameter, and (a) the range of spatial correlation. Figs. 3 and 4 indicate the directional experimental variograms in directions $0^{\circ}, 45^{\circ}, 90^{\circ}$, $135^{\circ}, 180^{\circ}, 225^{\circ}, 270^{\circ}$ and $315^{\circ}$, respectively.

Table 3 shows the parameters of the fitted models to the variograms constructed. All the variograms were fitted to spherical model. The variogram of 0 -direction shows significant difference in range which may demonstrate the enhancement of the variogram's spatial continuity. All variograms have low nugget values which indicate that the variables under study have strong spatial dependence. The very low $k$ values for all directions indicate a large lag $h$ and the sampling density closely matched the spatial variation. The global variogram can be written as:

$$
\gamma(h)= \begin{cases}0.0015+0.071\left(\frac{3 h}{5.1}-\frac{1}{2}\left(\frac{h}{3}\right)^{3}\right) & \text { for } h \leq 2.55 \\ 0.0725 & \text { for } h>2.55\end{cases}
$$

\subsection{Cross Validation and Interpretation of Results}

The reliability of the techniques was assessed by applying a cross validation technique. This allows comparing estimated and true values only using the information available in the known data set. In cross validation the estimation method is tested at the locations of existing samples. The value at a particular location was temporarily discarded from the sample data set; the value at the same location was then estimated using the remaining samples. Once the estimate was calculated it was compared to the true sample value that was initially removed. This procedure was repeated for all available samples. The resulting true and estimated values were compared using summary statistics like mean, standard deviation, and so on.

The cross validation results of the spherical model is shown in Table 3. The model has a mean error (ME) of -0.0015 , (which is very near to zero); mean square error (MSE) of 0.063 , (which is very low as 


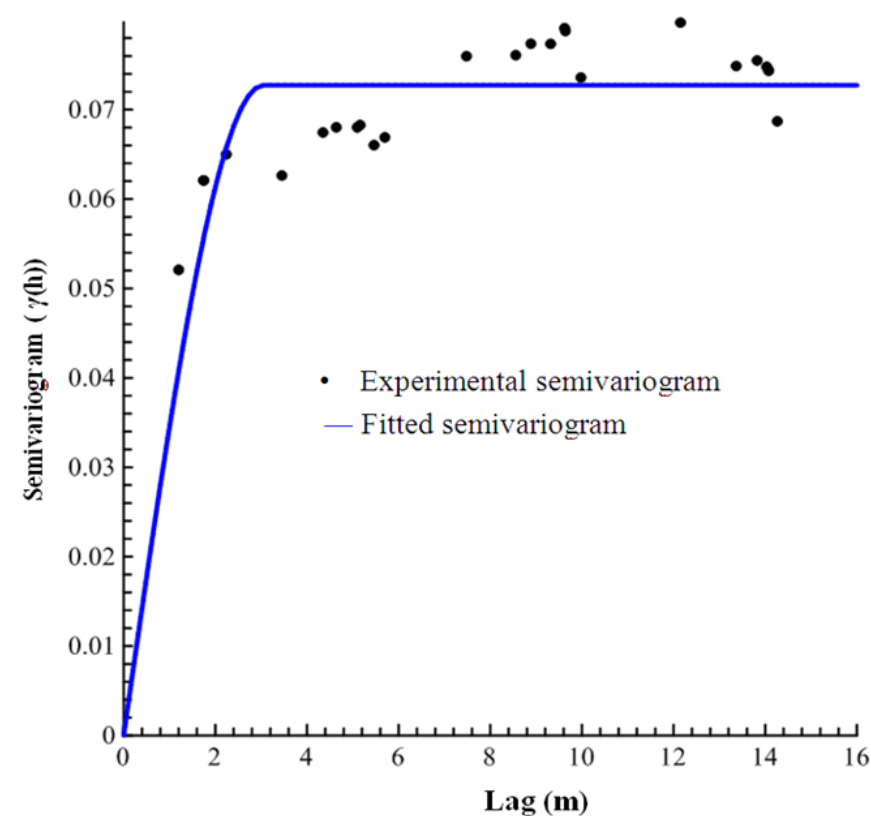

(a)

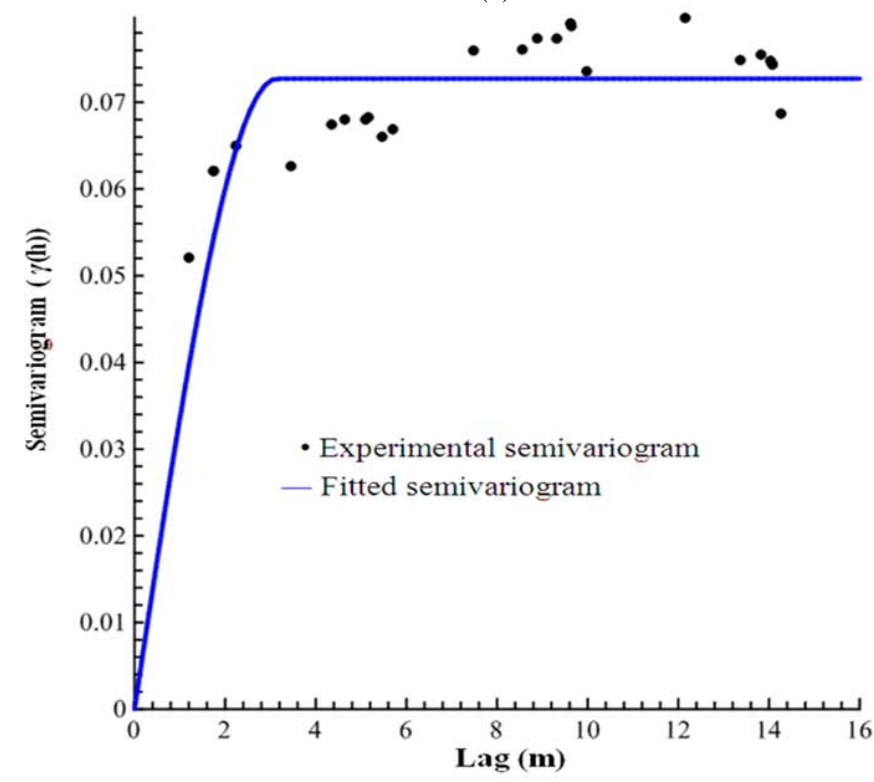

(c)

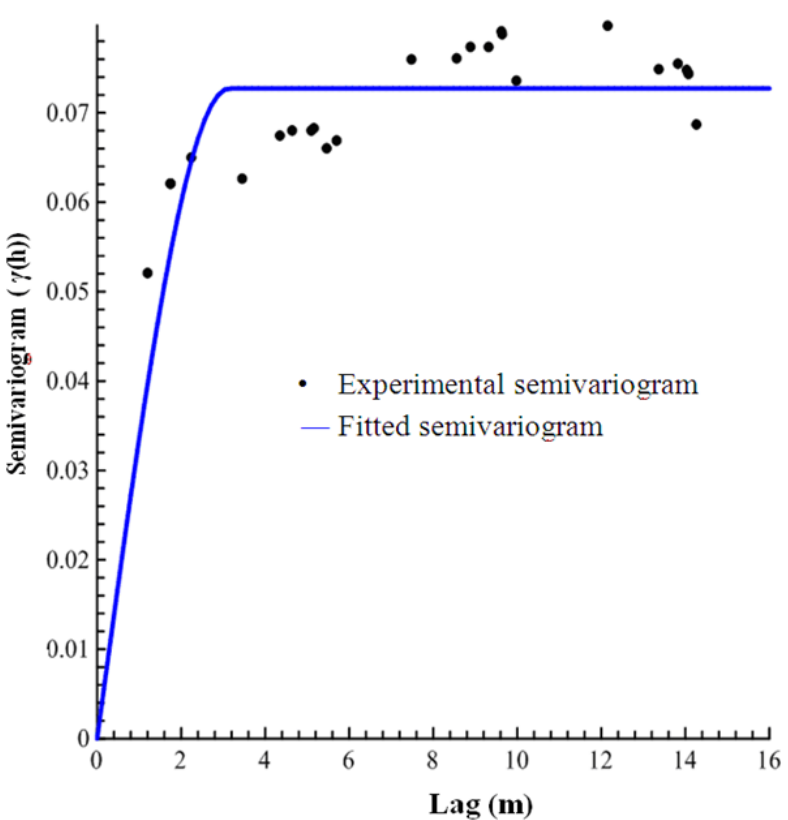

(b)

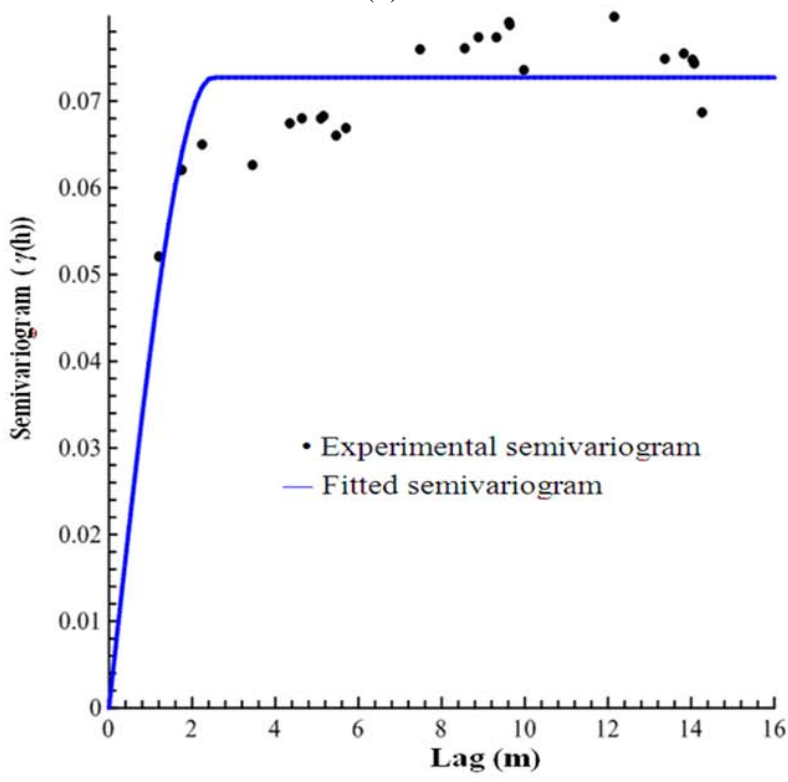

(d)

Fig. 3 Experimental and fitted semivariograms for steel oil storage tank thickness in different directions, (a) $0.0^{\circ}$, (b) $45^{\circ}$, (c) $90^{\circ}$ and (d) $135^{\circ}$.

compared to the variance of the data). Here the bracketed quantities refer to the requirements to consider a model as adequate [7]. The above cross validation results show that the chosen model and its parameters are adequate.

\subsection{Spatial Prediction}

Kriging was used to provide 2-D mapping of the thickness measurements. Kriging involves interpolation between measurement locations. Ordinary kriging (OK), which allows the mean of the measurements to vary spatially, was used in this study. This means that the kriged output provides a smoothed interpolation of the tank thickness since kriging predictions are weighted moving averages of the available data set $[15,16]$. The kriging thickness 
map is shown in Fig. 5. Ordinary kriging, always results in values that are "best" in the sense that the expected squared prediction error is minimal. However, the problem is that the field with predicted values is usually smoother than the field from which observations were obtained [15, 16].

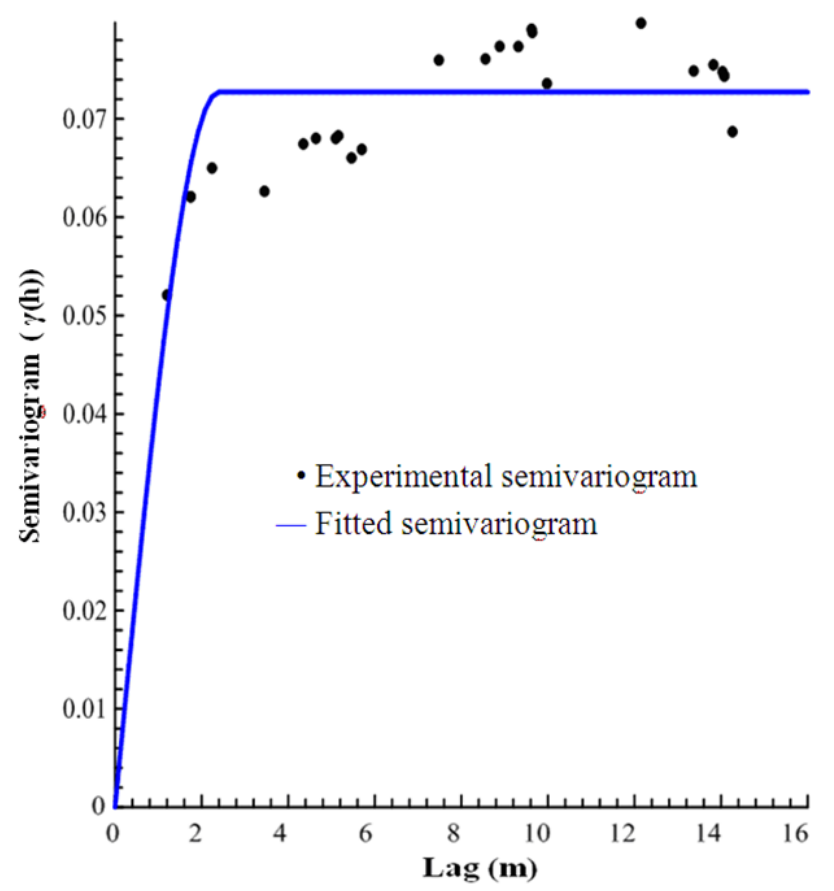

(a)

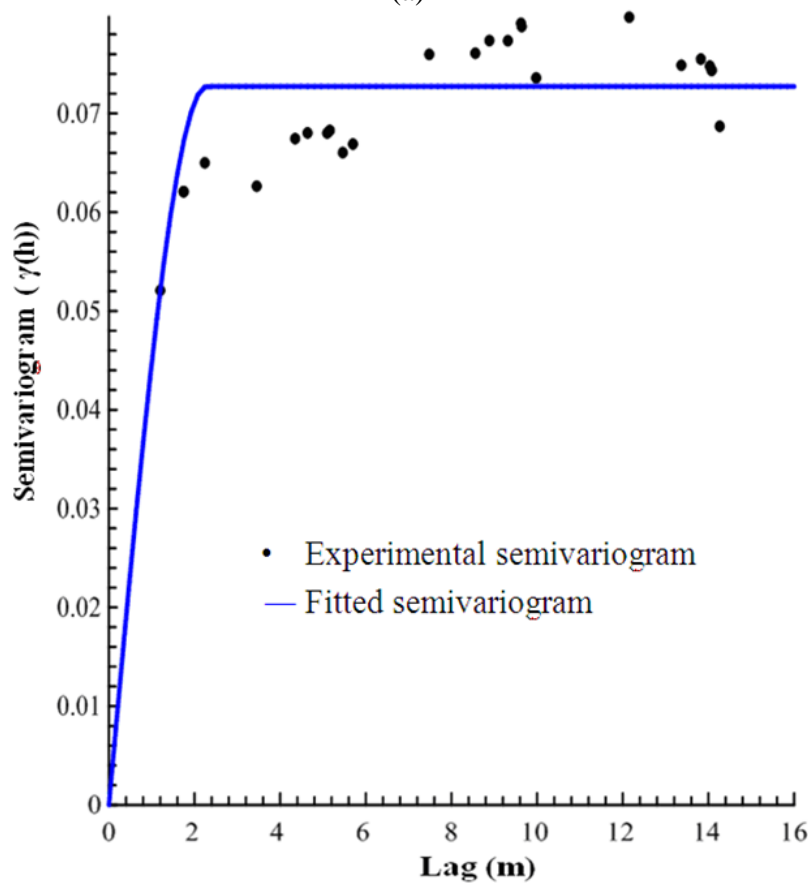

(c)
Recently, sequential simulation was proposed to overcome the limitations inherent in ordinary kriging. The simulation approach takes into account not only the spatial variation of observed data at sampled locations but also the variation in estimations at unsampled locations, which kriging estimation ignores

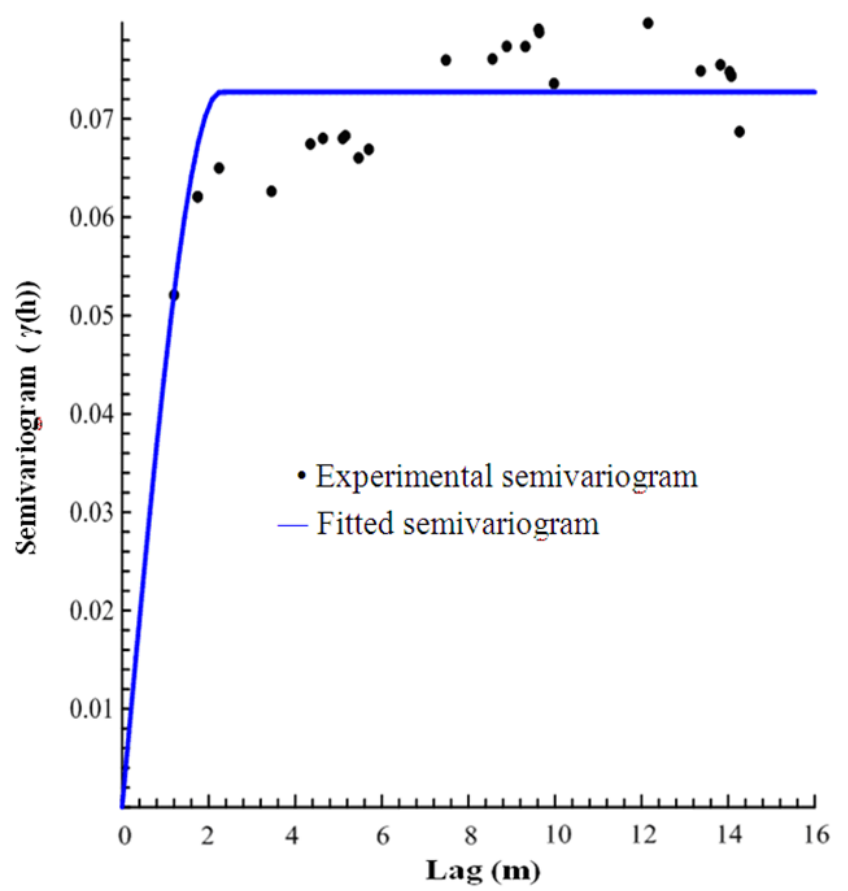

(b)

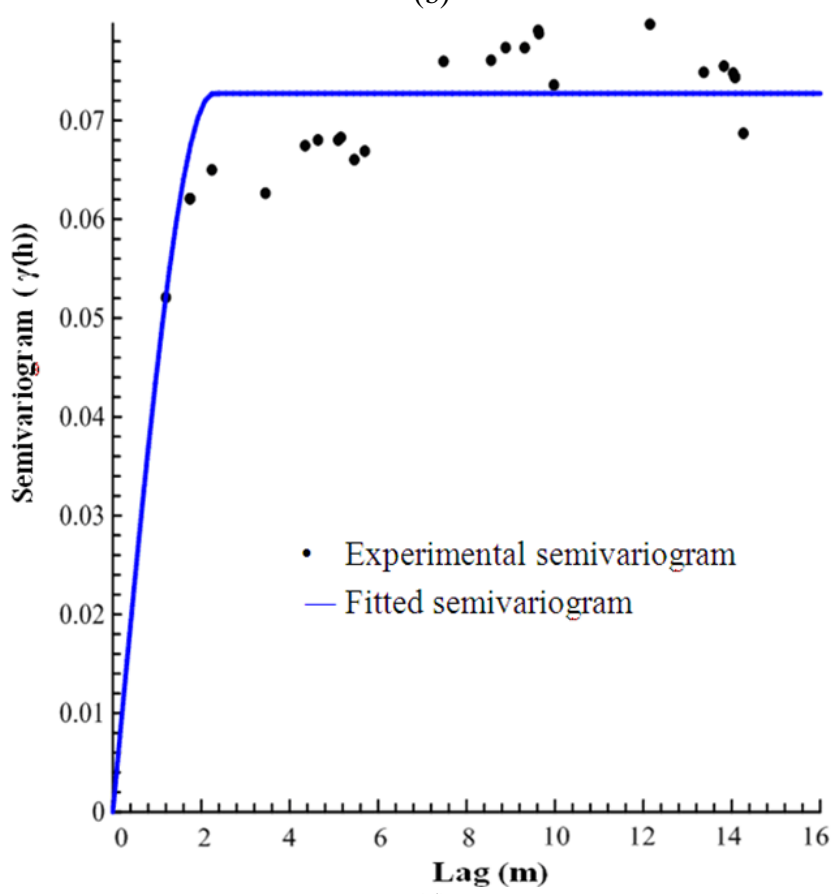

(d)

Fig. 4 Experimental and fitted semivariograms for steel oil storage tank thickness in different directions, (a) $180^{\circ}$, (b) $225^{\circ}$, (c) $270^{\circ}$ and (d) $315^{\circ}$. 
Table 2 Parameters of the spherical semivariogram models used.

\begin{tabular}{llllll}
\hline Direction & Semivariogram model type & Range (a) & Sill $\left(\mathrm{C}_{0}+\mathrm{C}\right)$ & Nugget $\mathrm{C}_{0}$ & ${\text { Ratio } \mathrm{k} \mathrm{C} /\left(\mathrm{C}_{0}+\mathrm{C}\right)}$ \\
\hline 0 & Spherical & 3.14 & 0.072 & 0.001 & 0.014 \\
45 & Spherical & 2.68 & 0.072 & 0.001 & 0.014 \\
90 & Spherical & 2.68 & 0.073 & 0.002 & 0.027 \\
135 & Spherical & 2.69 & 0.073 & 0.002 & 0.027 \\
180 & Spherical & 2.39 & 0.072 & 0.002 & 0.028 \\
225 & Spherical & 2.28 & 0.072 & 0.002 & 0.028 \\
270 & Spherical & 2.28 & 0.073 & 0.001 & 0.014 \\
315 & Spherical & 2.27 & 0.073 & 0.001 & 0.014 \\
\hline
\end{tabular}

Table 3 Regional-scale validation, performance obtained in cross-validation mode for the whole study tank.

\begin{tabular}{ll}
\hline Statistical factors & Thickness $(\mathrm{mm})$ \\
\hline Minimum & 13.9 \\
25th quartile & 14.4 \\
Median & 14.5 \\
75th quartile & 14.6 \\
Mean & 14.49 \\
Maximum & 14.72 \\
Coefficient of skewness & -0.69 \\
Coefficient of variation & 0.012 \\
ME & -0.0015 \\
MSE & 0.063 \\
\hline
\end{tabular}

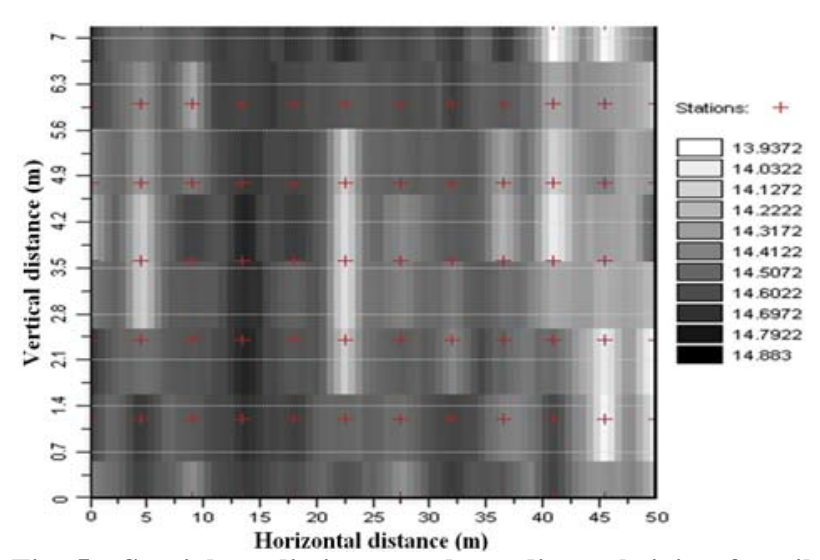

Fig. 5 Spatial prediction map by ordinary kriging for oil storage tank thickness.

$[11,17]$. Assessment of uncertainty for all data available in the geostatistical simulation is possible. All possible data at unsampled locations can be modeled. The simulation approach overcomes the failure of ordinary kriging to reproduce clusters. In addition, geostatistical simulations can collect mapping uncertainties at several locations simultaneously (i.e., multi-location uncertainty or spatial uncertainty) which is lacking in the ordinary kriging estimation used

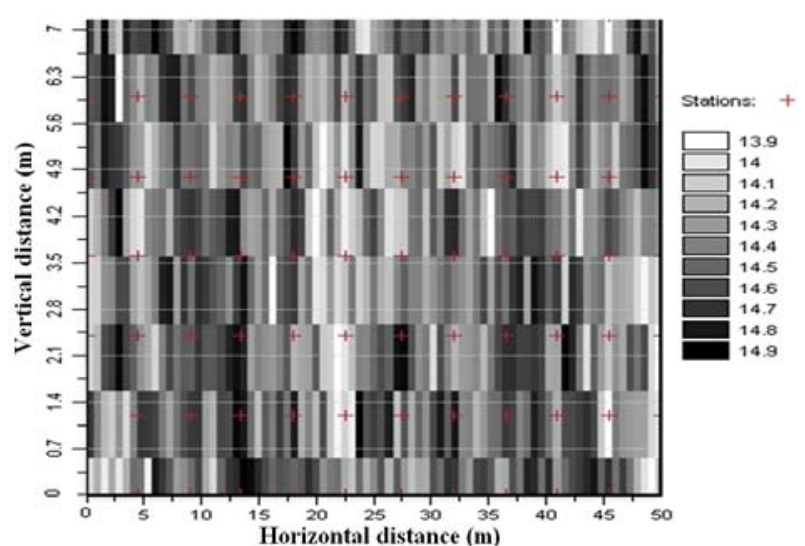

Fig. 6 Spatial prediction map by sequential simulation for oil storage tank thickness.

to assess the reliability [17-21]. Therefore, based on the semivariogram model, sequential simulation (Fig. 6) was used to generate a number of sets of values (realizations) that aim to reproduce each sample semivariogram and the theoretical semivariogram model. The technique used collected data to calculate the thicknes at unsampled locations. The simulation thickness map is shown in Fig. 6.

\section{Conclusions}

(1) Geostatistical analysis can provide necessary information for determining the spatial extend of inspection or the optimal number of samples that should be taken. It allows predicting the thickness for the required life along the tank. This information can facilitate the optimization of repair or maintenance strategies for oil steel tank structures;

(2) Characterization of spatial dependence by estimating a regular and theoretical semi-variograms indicated that the spherical model was generally the 
best to describe the tank thickness;

(3) The approach with spatial variability provides more differentiated information about the condition of the tank and so the service life time can be predicted more accurately.

\section{References}

[1] M.M. Sadawy, R.Q. Heseinov, T.I. Shirinov, Corrosion and electrochemical behavior of austenitic-ferretic stainless steel in sulfuric acid solutions Geochem. Problems and Chem. Baku (2009) 327-332.

[2] S. Kessler, M. Huber, D. Straub, C. Gehlen, C. Moormann, Stochastic evaluation of active corroding areas in concrete structures, in: Proceedings of the 8th International Probabilistic Workshop, Szczecin, 2010, pp. $1-13$.

[3] D. Li, S. Zhang, W. Yang, W. Zhang, Corrosion monitoring and evaluation of reinforced concrete structures utilizing the ultrasonic guided wave technique, International Journal of Distributed Sensor Networks, Vol. (2014) 1-9

[4] R.M. Lark, Optimized spatial sampling of soil for estimation of the variogram by maximum likelihood, Geoderma 105 (2002) 49-80.

[5] F. Kuang, J. Zhang, C. Zou, T. Shi, Y. Wang, Shihong Zhang, H. Xu, Electrochemical methods for corrosion monitoring: a survey of recent patents, Recent Patents on Corrosion Science 2 (2010) 34-39

[6] K. Murthy, G. Abbaiah,Geostatistical analysis for estimation of mean rainfalls in Andhra Pradesh, India, Inter. J. Geology 1 (3) (2007) 35-51.

[7] S. Ly, C. Charles, A. Degr, Geostatistical interpolation of daily rainfall at catchment scale: the use of several variogram models in the Ourthe and Ambleve catchments, Belgium, Hydrol. Earth Syst. Sci. 15 (2011) 2259-2274.

[8] V. Kumar, Remadevi, Kriging of groundwater levels: A case study, JOSH 6 (2006) 81-94.

[9] R.A. Taany, B. Tahboub, G.A. Saffarini, Geostatistical analysis of spatiotemporal variability of groundwater level fluctuations in Amman-Zarqa basin, Jordan: A case study, Environ. Geo. 157 (2009) 525-535.
[10] P.N. Nouck, C. Kenfack, A.D. Diab, K. Njeudjang, L. Jorelle, R. Kamseu, A geostatistical re-interpretation of gravity surveys in the Yagoua, Cameroon region, Geofísica Internacional 52-4 (2013) 365-373.

[11] A.F. Corte, J.M. Calaforra, R. J.Espinos, F.S. Martos, Geostatistical spatiotemporal analysis of air temperature as an aid to delineating thermal stability zones in a potential show cave: Implications for environmental management. Jour. of Envir. Manag. 81 (2006) 371-383.

[12] K. Hemker, E. Berg, M. Bakker, Ground water whirls, Ground Water 42 (2) (2004) 234-242.

[13] M. Gilbert, J. Claude, Site condition and predation influence a bark beetle's success: A spatially realistic approach, Agricu. Forest Entom. 5 (2) (2003) 87-96.

[14] C.D. Lloyd, P.M. Atkinson, Archaeology and geostatistics, Jour. of Archaeo. Scie. 31 (2004) 151-165.

[15] C. Prudhomme, D.W. Reed, Mapping extreme rainfall in a mountainous region using geostatistical techniques: A case study in Scotland, Int. J. Climatol. 19 (1999) 1337-1356.

[16] P. Goovaerts, Geostatistics for Natural Resources Evaluation, Oxford University Press, New York, 1997, pp. 187-233.

[17] E. Mapfumo, D.S Chanasyk, C.L.A. Chaikowsky, Stochastic simulation of soil water status on reclaimed land in northern Alberta, JOSH 6 (2006) 34-44.

[18] U.S. Environmental Protection Agency, Developing spatially interpolated surfaces and estimating uncertainty, Research Triangle Park NC 27711 (2004) 1-159.

[19] A. Hamanna, M.P. Koshya, G. Namkoonga, C.C. Ying, Genotype $\mathrm{x}$ environment interactions in Alnus rubra: Developing seed zones and seed-transfer guidelines with spatial statistics and GIS, Forest Ecology and Manag. 136 (2000) 107-119.

[20] K.W. Juang, Y.S. Chen, D.Y. Lee, Using sequential indicator simulation to assess the uncertainty of delineating heavy-metal contaminated soils, Enviro. Pollution. 127 (2004) 229-238.

[21] J.M. Miras-Avalos, A.P. Gonzalez, E.V. Vazquez, P.S. Fouz, Mapping monthly rainfall data in Galicia (NW Spain) using inverse distances and geostatistical methods, Adv. Geosci. 10 (2007) 51-57. 